\title{
GMR
}

\section{Genetic control and combining ability of agronomic attributes and northern leaf blight-related attributes in popcorn}

J.S. Santos, A.T Amaral Júnior, M. Vivas, G.S. Mafra, G.F. Pena, F.H.L. Silva and A.G. Guimarães

Laboratório de Genética e Melhoramento Vegetal, Universidade Estadual do Norte Fluminense Darcy Ribeiro, Campos dos Goytacazes, RJ, Brasil

Corresponding author: J.S. Santos

E-mail: julianasaltiresdossantos@yahoo.com.br

Genet. Mol. Res. 16 (3): gmr16039772

Received July 6, 2017

Accepted August 25, 2017

Published September 27, 2017

DOI http://dx.doi.org/10.4238/gmr16039772

Copyright $(2017$ The Authors. This is an open-access article distributed under the terms of the Creative Commons Attribution ShareAlike (CC BY-SA) 4.0 License.

\begin{abstract}
The present study was conducted to investigate the genetic control and to estimate the general and specific combining abilities of popcorn for agronomic attributes and attributes related to resistance to northern leaf blight (NLB). The 56 hybrids ( $F_{1}$ and reciprocals), together with the eight parent lines and six controls, were evaluated in two harvests, in a randomized-block design with four replications. Dominance components were more expressive than the additive components for grain yield and expression of resistance, and hybridization was the most suitable option for obtaining resistant and productive genotypes. For grain yield, popping expansion, and resistance to NLB, there was no significance for reciprocal effects, which indicates that the direction in which the cross is performed does not interfere with the hybrid's performance. Then, the superior hybrids recommended for more profitable growth were P8 $\mathrm{x}$ L61, L61 x L76, and L61 x L77.
\end{abstract}

Key words: Zea mays; Genetic resistance; Leaf disease; Diallel analysis 


\section{INTRODUCTION}

Popcorn (Zea mays L.) is a type of corn whose main characteristic is its hard and small kernels, which hold the ability to expand as a result of an internal pressure when heated (Hoseney et al., 1983; Silva et al., 1993). Typically, when compared with cultivars of common corn, popcorn plants display a greater susceptibility to the attack of pests and diseases (Hallauer, 2001; Arnhold, 2008; Leonello et al., 2009). Besides contributing to low yields, this fact elevates production risks. Therefore, producing genotypes resistant to the main leaf diseases should be considered a relevant aspect in breeding programs for this species (Arnhold, 2008).

Northern leaf blight (NLB), a disease caused by Exserohilum turcicum (Pass.) Leonard and Suggs (sin. Helminthosporium turcicum Pass.), is widespread all over the world and practically in all corn grown (Smith and White, 1988; Carson, 1995). In popcorn, in particular, it is considered one of the main leaf diseases (Fantin et al., 1991; Miranda et al., 2002; Sabato and Pinto, 2013). The pathogen causes losses that can exceed $40 \%$ of the grain yield under favorable climatic conditions and in susceptible genotypes. In Brazil, the greatest severities of diseases have occurred in off-season crops, when the pathogen infects the plants during the flowering period (Costa et al., 2009). Because of the growing participation of the off-season seasons for this species, it is even more important to control diseases of higher incidence during this growing season.

Several control measures are implemented to minimize the damage caused by NLB (spot blotch), e.g., the spraying of fungicides and the planting of resistant varieties. Furthermore, some practices are adopted, such as crop rotation, the use of the adequate planting density and spacing, and the use of balanced fertilization and elimination of crop residues (Bergamin Filho and Amorim, 2011). Of the recommended measures, the use of resistant cultivars is the most effective means of control (Ishfaq et al., 2014), as it decreases production costs and reduces the risks to the activity of man and the environment (Vieira et al., 2009).

According to Paterniani and Miranda Filho (1978) and Hallauer et al. (2010), the breeding of corn has two alternatives that can be implemented together: obtaining genetically improved populations or hybrids. In the first case, by using adequate selection methods, the frequency of favorable genes in the enriched population can be gradually increased. In the second scenario, the breeding strategy is aimed at producing inbred lines that, when in proper combinations, can produce hybrids superior to the populations of origin. In the conception of Cruz et al. (2012), in the case of hybrid production, the diallel analysis has been exploited successfully, as it provides an estimate of useful parameters in the selection of parents for hybridization in a simple manner, as well as information about promising combinations.

Despite the advantages of diallel-cross strategies, however, little research has been conducted with popcorn using this methodology. The studies undertaken by Larish and Brewbaker (1999), Pinto et al. (2007), Pajic et al. (2008), da Silva et al. (2010), Viana et al. (2011), Vieira et al. (2011), Moterle et al. (2012), and Cabral et al. (2015) are the few examples in which diallels were used in popcorn, in which lines are used as parents. In this group, Vieira et al. (2011) evaluated grain yield (GY), popping expansion (PE), and partial resistance to southern corn rust (Puccinia polysora Underw) in hybrids originating from crosses in a partial circulating diallel scheme among ten popcorn lines (IAC 112 line groups $\mathrm{x}$ 'Zaeli' line groups). The authors observed a significant effect of general combining ability (GCA) in the Zaelin group for GY, PE, and resistance to southern corn rust and concluded that there is a

Genetics and Molecular Research 16 (3): gmr16039772 
predominance of additive genes in the expression of resistance, and these recurrent selection methods are recommended for obtaining gains in these traits.

At the moment, there are no studies on resistance to NLB employing diallel analysis in popcorn, although different levels of resistance to the disease have been reported in evaluations of popcorn genotypes (Fantin et al., 1991; Miranda et al., 2002; Vieira et al., 2009). Given the considerations mentioned above, the present study aimed to evaluate the genetic effects and the combining ability of hybrids of popcorn lines for incidence and severity of NLB, as well as for GY and PE, via diallel analysis, in the first and second harvests.

\section{MATERIAL AND METHODS}

Single-cross hybrids were produced, and parents and $\mathrm{F}_{1}$ hybrids with reciprocals were evaluated at the Antônio Sarlo State Agricultural College, located in Campos dos Goytacazes - RJ, Brazil. The parent lines and diallel hybrids were cultivated in the agricultural periods of the first harvest (October 2014 to January 2015) and the second harvest (May to August 2015). The trial consisted of 70 treatments, including 56 simple hybrids ( $F_{1}$ and reciprocals), eight parents ( $\mathrm{S}_{7}$ lines), and six controls, which were selected according to their performance regarding agronomic attributes and resistance to spot blotch, aiming to compare these traits in the controls with the hybrids. The adopted controls were IAC 125, BRS Angela, UENF 14, UFV M2-Barão de Viçosa, and hybrids L70 x L54 and P8 x L54.

The trials were implemented in a randomized-block design with four replications. The lines were chosen at random and separated from the hybrids to prevent competition effects. Plots consisted of a $5.0-\mathrm{m}$ planting row with 25 plants, with $0.90 \mathrm{~m}$ spacing between rows and $0.20 \mathrm{~m}$ between plants. Grains were seeded at a depth of $0.05 \mathrm{~m}$, using three grains per furrow; 30 days later, the area was thinned, leaving one plant per furrow. Plots were fertilized at seeding with $60 \mathrm{~kg} / \mathrm{ha} \mathrm{K}_{2} \mathrm{O}, 30 \mathrm{~kg} / \mathrm{ha} \mathrm{N}$, and $60 \mathrm{~kg} / \mathrm{ha} \mathrm{P}_{2} \mathrm{O}_{5}$, in addition to $100 \mathrm{~kg} / \mathrm{ha} \mathrm{N}$. Irrigation was applied by a sprinkling system, and herbicides and insecticides were applied whenever necessary.

To evaluate the incidence and severity of NLB, caused by E. turcicum, five plants were analyzed per plot. The assessments took place during the flowering period and the grain's dough stage. Three evaluations were carried out, once a week. To study the incidence of $E$. turcicum in the plant, the scores proposed by Agroceres (1996) were adopted, and then, the proportion of leaf area of the plant was evaluated, considering all leaves. The severity of the disease in the leaf was determined by using a diagrammatic scale proposed by Vieira et al. (2013). In the study of severity in the leaf, only the leaf of the uppermost cob of the plant was considered.

Further, GY and PE were evaluated. The former was determined by weighing the grains after eliminating the cob, relative to the area extrapolated to hectares, and was expressed as $\mathrm{kg} /$ ha. To determine the $\mathrm{PE}(\mathrm{mL} / \mathrm{g})$, the weight of $30 \mathrm{~g}$ grains was heated in a microwave oven inside a special bag for popping at the power of $1000 \mathrm{~W}$ for $2 \mathrm{~min}$ and $20 \mathrm{~s}$. The popcorn volume was quantified in a $2000-\mathrm{mL}$ beaker, by dividing the popped volume by 30 (weight of grains).

The data obtained from the experimental plots were initially subjected to an individual analysis of variance for each location, and combined analysis of variance in a completely randomized block design. Whenever a significant effect was detected, treatment means were grouped by the Scott-Knott algorithm at the $5 \%$ probability level.

In the diallel analysis, we used Griffing's (1956) Method 1, Model B. The GCA effects $\left(\hat{\mathrm{g}}_{\mathrm{i}}\right)$ of each parent and the specific combining ability (SCA) effect $\left(\hat{\mathrm{s}}_{\mathrm{ij}}\right)$ from a set of parental $(\mathrm{p})$

Genetics and Molecular Research 16 (3): gmr16039772 
and $[p(p-1) / 2] F_{1}$ hybrids with the reciprocals were estimated. All analyses were performed using the computer resources of the Genes software (Cruz, 2013).

\section{RESULTS AND DISCUSSION}

For both incidence and severity of E. turcicum, there were significant effects for all tested sources of variation (data not shown); it thus follows that there is variation between the treatments, which allows us to anticipate the opportunity to identify lines and hybrids of interest for obtaining selective gains. A significant effect of growing harvest was already expected, given that the development of the disease is influenced by environmental variations, e.g., temperature and relative humidity of the air (Agrios, 1988). Costa et al. (2009) reported that the disease is more severe in the conditions of the second harvest, which is in line with the results obtained in the present study.

Considering the occurrence of a significant treatment $\mathrm{x}$ harvest interaction effect, individual analyses were performed for each growing harvest. A significant effect of treatment was observed in both harvests, indicating the existence of genetic variation, and consequently, the possibility of selection of superior genotypes. The variation of treatments as observed by the F-test was also detected by the Scott-Knott algorithm, and then, three (for severity in the first harvest) and six (for incidence in the second harvest) groups were formed (Table 1).

The group of genotypes that showed the lowest mean values for incidence and severity in both growing harvests, and consequently, the highest resistance levels was formed by parent L76, control UFV M2-Barão de Viçosa, and the combinations L55 x L61, L55 x L70, L55 x L76, L55 x L88, L55 x P8, L61 x L76, L61 x L77, L70 x L55, L70 x L88, L76 x L77, L77 x L76, L88 x L77, L88 x L61, L88 x L70, L88 x L76, L88 x P8, P8 x L61, and P8 x L88 (Table 1). According to the genealogy of the hybrids mentioned above, it is observed that the 18 combinations included lines L88, L77, L55, and L76 in their constitution that were obtained from varieties Viçosa and Beija-Flor, and were considered potential sources of resistance to NLB caused by E. turcicum (Miranda et al., 2003). The same was true for lines L70 and L61, which originated from cultivar BRS Angela, which has been reported as moderately resistant to E. turcicum (Embrapa, 2008). In this case, it was inferred that the lines provided experimental hybrids with the genetic contribution that culminated in higher levels of resistance in hybrids originating from lines of variety Beija-Flor. Therefore, the use of these parents in crosses tends to benefit the introduction of resistance genes, thereby making them of interest for breeding.

For grain yield, the means of the genotypes in the first harvest were allocated into three groups, whereas four groups were formed in the second harvest. In the two growing harvests, good performance was shown by the hybrids, since the means expressed by the genotypes that made up the group with the highest yields exceeded the magnitude of 3000.0 $\mathrm{kg} / \mathrm{ha}$. Of the genotypes that showed, in the first harvest, the best performance for grain yield, hybrids P8 x L61, L61 x L88, L77 x L55, and L88 x L77 stood out with estimated yields of $4767.01,4648.00,4502.03$, and $4495.34 \mathrm{~kg} / \mathrm{ha}$, respectively. We also observed that among the lowest magnitudes for grain yield were varieties BRS Angela and UFV-M2 Barão de Viçosa, with mean values of 2560.12 and $2685.74 \mathrm{~kg} / \mathrm{ha}$, respectively. Therefore, these controls did not adapt well to Campos dos Goytacazes in the first growing harvest. In the second harvest, 20 genotypes expressed mean values for grain yield between 4197.92 and $5411.23 \mathrm{~kg} / \mathrm{ha}$. The highest mean for this trait was obtained in the second harvest. The experimental hybrids with the highest means for grain yield were L77 x L55, L61 x L77, L61 x L55, L77 x P1, with estimated yields of $5411.23,4969.94,4946.64$, and $4787.83 \mathrm{~kg} / \mathrm{ha}$, respectively.

Genetics and Molecular Research 16 (3): gmr16039772 
Table 1. Means clustering test for incidence and severity of northern leaf blight (NLB) evaluated in 8 parents, 56 hybrids, and 6 controls in the first harvest (October 2014 to January 2015) and the second harvest (May to August 2015) in Campos dos Goytacazes, RJ, Brazil.

\begin{tabular}{|c|c|c|c|c|c|c|c|c|}
\hline \multirow[t]{2}{*}{ Genotypes } & \multicolumn{2}{|c|}{ Incidence of NLB } & \multicolumn{2}{|c|}{ Severity of NLB } & \multicolumn{2}{|c|}{ Grain yield $(\mathrm{kg} / \mathrm{ha})$} & \multicolumn{2}{|c|}{ Popping expansion $(\mathrm{mL} / \mathrm{g})$} \\
\hline & 1st harvest & 2 nd harvest & 1st harvest & 2 nd harvest & 1st harvest & 2 nd harvest & 1st harvest & 2 nd harvest \\
\hline L55xL61 & $11.85 \mathrm{c}$ & $10.69 \mathrm{f}$ & $11.85 \mathrm{c}$ & $1.65 \mathrm{~d}$ & $4,343.91 \mathrm{a}$ & $3,674.22 \mathrm{~b}$ & $26.34 \mathrm{c}$ & $27.75 \mathrm{a}$ \\
\hline L55xL70 & $9.56 \mathrm{c}$ & $9.99 \mathrm{f}$ & $9.56 \mathrm{c}$ & $1.49 \mathrm{~d}$ & $3,504.58 \mathrm{~b}$ & $3,743.56 \mathrm{~b}$ & $26.13 \mathrm{c}$ & $24.84 \mathrm{~b}$ \\
\hline L55xL76 & $6.77 \mathrm{c}$ & $11.30 \mathrm{f}$ & $6.77 \mathrm{c}$ & $0.65 \mathrm{~d}$ & $3,613.17 \mathrm{~b}$ & $4,716.69 \mathrm{a}$ & $30.17 \mathrm{~b}$ & $21.09 \mathrm{c}$ \\
\hline L55xL77 & $13.76 \mathrm{c}$ & $17.42 \mathrm{e}$ & $13.76 \mathrm{c}$ & $3.37 \mathrm{~d}$ & $3,470.24 \mathrm{~b}$ & $4,621.03 \mathrm{a}$ & $26.25 \mathrm{c}$ & $24.26 \mathrm{~b}$ \\
\hline L55xL88 & $13.26 \mathrm{c}$ & $5.26 \mathrm{f}$ & $13.26 \mathrm{c}$ & $0.13 \mathrm{~d}$ & $4,210.48 \mathrm{a}$ & $4,544.87 \mathrm{a}$ & $24.71 d$ & $20.67 \mathrm{c}$ \\
\hline L55xP1 & $10.44 \mathrm{c}$ & $21.94 \mathrm{e}$ & $10.44 \mathrm{c}$ & $3.88 \mathrm{~d}$ & $3,744.33 \mathrm{~b}$ & $3,130.30 \mathrm{c}$ & $25.59 \mathrm{c}$ & $26.17 \mathrm{~b}$ \\
\hline L55xP8 & $9.64 \mathrm{c}$ & $10.14 \mathrm{f}$ & $9.64 \mathrm{c}$ & $2.08 \mathrm{~d}$ & $4,561.11 \mathrm{a}$ & $3,463.94 \mathrm{~b}$ & $30.63 \mathrm{~b}$ & $25.25 \mathrm{~b}$ \\
\hline L61xL55 & $18.95 \mathrm{~b}$ & $9.32 \mathrm{f}$ & $18.95 \mathrm{~b}$ & $0.47 \mathrm{~d}$ & $3,645.64 \mathrm{~b}$ & $4,946.64 \mathrm{a}$ & $27.17 \mathrm{c}$ & $30.00 \mathrm{a}$ \\
\hline L61xL70 & $16.37 \mathrm{c}$ & $10.48 \mathrm{f}$ & $16.37 \mathrm{c}$ & $1.81 \mathrm{~d}$ & $4,313.39 \mathrm{a}$ & $3,828.60 \mathrm{~b}$ & $25.46 \mathrm{c}$ & $31.17 \mathrm{a}$ \\
\hline L61xL76 & $7.58 \mathrm{c}$ & $8.30 \mathrm{f}$ & $7.58 \mathrm{c}$ & $1.15 \mathrm{~d}$ & $4,118.21 \mathrm{a}$ & $4,492.78 \mathrm{a}$ & $26.71 \mathrm{c}$ & $29.63 \mathrm{a}$ \\
\hline L61xL77 & $12.93 \mathrm{c}$ & $11.94 \mathrm{f}$ & $12.93 \mathrm{c}$ & $1.73 \mathrm{~d}$ & $4,311.84 \mathrm{a}$ & $4,969.94 \mathrm{a}$ & $27.25 \mathrm{c}$ & $31.50 \mathrm{a}$ \\
\hline L61xL88 & $18.36 \mathrm{~b}$ & $8.23 \mathrm{f}$ & $18.36 \mathrm{~b}$ & $0.32 \mathrm{~d}$ & $4,648.00 \mathrm{a}$ & $4,380.38 \mathrm{a}$ & $21.88 \mathrm{e}$ & $24.42 \mathrm{~b}$ \\
\hline L61xP1 & $21.73 \mathrm{~b}$ & $21.14 \mathrm{e}$ & $21.73 \mathrm{~b}$ & $1.76 \mathrm{~d}$ & $3,742.28 \mathrm{~b}$ & $3,614.79 b$ & $25.54 \mathrm{c}$ & $31.00 \mathrm{a}$ \\
\hline L61xP8 & $14.87 \mathrm{c}$ & $20.97 \mathrm{e}$ & $14.87 \mathrm{c}$ & $4.60 \mathrm{~d}$ & $4,254.48 \mathrm{a}$ & $3,681.30 \mathrm{~b}$ & $30.73 b$ & $31.83 \mathrm{a}$ \\
\hline L70xL55 & $7.57 \mathrm{c}$ & $10.69 \mathrm{f}$ & $7.57 \mathrm{c}$ & $1.16 \mathrm{~d}$ & $4,018.89 \mathrm{a}$ & $4,197.92 \mathrm{a}$ & $27.46 \mathrm{c}$ & $23.34 \mathrm{~b}$ \\
\hline L70xL61 & $17.22 \mathrm{c}$ & $17.24 \mathrm{e}$ & $17.22 \mathrm{c}$ & $3.69 \mathrm{~d}$ & $3,879.00 \mathrm{a}$ & $3,940.50 \mathrm{~b}$ & $27.38 \mathrm{c}$ & $30.75 \mathrm{a}$ \\
\hline L70xL76 & $8.29 \mathrm{c}$ & $28.68 \mathrm{e}$ & $8.29 \mathrm{c}$ & $5.60 \mathrm{~d}$ & $3,909.59 \mathrm{a}$ & $3,734.11 \mathrm{~b}$ & $30.46 \mathrm{~b}$ & $26.00 \mathrm{~b}$ \\
\hline L70xL88 & $13.42 \mathrm{c}$ & $7.63 \mathrm{f}$ & $3.10 \mathrm{c}$ & $0.73 \mathrm{~d}$ & $3,553.60 \mathrm{~b}$ & $3,666.81 \mathrm{~b}$ & $28.46 \mathrm{c}$ & $22.00 \mathrm{c}$ \\
\hline L70xL77 & $10.88 \mathrm{c}$ & $23.56 \mathrm{e}$ & $4.87 \mathrm{~b}$ & $5.22 \mathrm{~d}$ & $3,545.45 \mathrm{~b}$ & $4,286.40 \mathrm{a}$ & $26.50 \mathrm{c}$ & $30.25 \mathrm{a}$ \\
\hline L70xP1 & $19.92 \mathrm{~b}$ & $21.71 \mathrm{e}$ & $9.52 \mathrm{~b}$ & $6.69 \mathrm{c}$ & $2,038.37 \mathrm{c}$ & $2,943.92 \mathrm{c}$ & $28.46 \mathrm{c}$ & $30.56 a$ \\
\hline L70xP8 & $14.98 \mathrm{c}$ & $23.64 \mathrm{e}$ & $5.29 \mathrm{~b}$ & $7.03 \mathrm{c}$ & $3,611.88 \mathrm{~b}$ & $2,536.97 \mathrm{c}$ & $33.87 \mathrm{a}$ & $30.59 \mathrm{a}$ \\
\hline L76xP1 & $10.93 \mathrm{c}$ & $21.01 \mathrm{e}$ & $2.70 \mathrm{c}$ & $2.82 \mathrm{~d}$ & $4,089.00 \mathrm{a}$ & $4,390.84 \mathrm{a}$ & $30.96 \mathrm{~b}$ & $25.75 \mathrm{~b}$ \\
\hline L76xL55 & $11.60 \mathrm{c}$ & $19.37 \mathrm{e}$ & $1.32 \mathrm{c}$ & $1.80 \mathrm{~d}$ & $3,097.46 \mathrm{~b}$ & $4,485.45 \mathrm{a}$ & $28.08 \mathrm{c}$ & $21.92 \mathrm{c}$ \\
\hline L76xL61 & $8.22 \mathrm{c}$ & $31.68 \mathrm{e}$ & $1.10 \mathrm{c}$ & $6.25 \mathrm{c}$ & $3,755.58 \mathrm{~b}$ & $3,732.35 \mathrm{~b}$ & $26.09 \mathrm{c}$ & $28.33 \mathrm{a}$ \\
\hline L76xL70 & $22.20 \mathrm{~b}$ & $52.33 \mathrm{c}$ & $4.18 \mathrm{c}$ & $10.85 \mathrm{~b}$ & $2,557.71 \mathrm{c}$ & $2,894.31 \mathrm{c}$ & $30.33 \mathrm{~b}$ & $26.25 \mathrm{~b}$ \\
\hline L76xL77 & $7.08 \mathrm{c}$ & $9.78 \mathrm{f}$ & $1.59 \mathrm{c}$ & $0.59 \mathrm{~d}$ & $3,871.41 \mathrm{a}$ & $3,898.32 \mathrm{~b}$ & $29.92 \mathrm{~b}$ & $22.08 \mathrm{c}$ \\
\hline L76xL88 & $14.76 \mathrm{c}$ & $50.50 \mathrm{c}$ & $3.58 \mathrm{c}$ & $10.32 \mathrm{~b}$ & $3,199.02 \mathrm{~b}$ & $3,524.20 \mathrm{~b}$ & $25.00 \mathrm{~d}$ & $19.09 \mathrm{~d}$ \\
\hline L76xP8 & $8.62 \mathrm{c}$ & $27.98 \mathrm{e}$ & $1.38 \mathrm{c}$ & $5.18 \mathrm{~d}$ & $3,290.18 \mathrm{~b}$ & $3,197.01 \mathrm{c}$ & $34.46 a$ & $28.83 \mathrm{a}$ \\
\hline L77xP1 & $11.30 \mathrm{c}$ & $17.56 \mathrm{e}$ & $3.84 \mathrm{c}$ & $2.56 \mathrm{~d}$ & $3,830.97 \mathrm{a}$ & $4,787.83 \mathrm{a}$ & $30.37 \mathrm{~b}$ & $30.84 \mathrm{a}$ \\
\hline $\begin{array}{l}\text { L77xL55 } \\
\end{array}$ & $12.03 \mathrm{c}$ & $16.57 \mathrm{e}$ & $1.40 \mathrm{c}$ & $2.20 \mathrm{~d}$ & $4,502.03 \mathrm{a}$ & $5,411.23 \mathrm{a}$ & $27.96 \mathrm{c}$ & $22.92 \mathrm{~b}$ \\
\hline L77xL61 & $10.53 \mathrm{c}$ & $26.04 \mathrm{e}$ & $1.91 \mathrm{c}$ & $5.05 \mathrm{~d}$ & $3,625.70 \mathrm{~b}$ & $4,210.63 \mathrm{a}$ & $25.21 \mathrm{~d}$ & $31.58 \mathrm{a}$ \\
\hline L77xL70 & $14.81 \mathrm{c}$ & $50.50 \mathrm{c}$ & $3.29 \mathrm{c}$ & $8.56 \mathrm{c}$ & $2,477.70 \mathrm{c}$ & $2,804.77 \mathrm{c}$ & $30.54 \mathrm{~b}$ & $30.08 \mathrm{a}$ \\
\hline L77xL76 & $7.74 \mathrm{c}$ & $5.32 \mathrm{f}$ & $1.21 \mathrm{c}$ & $0.35 \mathrm{~d}$ & $3,960.76 \mathrm{a}$ & $4,582.80 \mathrm{a}$ & $30.29 \mathrm{~b}$ & $25.42 \mathrm{~b}$ \\
\hline L77xL88 & $11.21 \mathrm{c}$ & $43.94 \mathrm{~d}$ & $3.69 \mathrm{c}$ & $7.52 \mathrm{c}$ & $3,246.53 \mathrm{~b}$ & $3,310.99 b$ & $26.71 \mathrm{c}$ & $17.70 \mathrm{~d}$ \\
\hline L77xP8 & $10.75 \mathrm{c}$ & $35.93 \mathrm{~d}$ & $1.63 \mathrm{c}$ & $6.93 \mathrm{c}$ & $3,758.75 b$ & $3,095.58 \mathrm{c}$ & $33.38 \mathrm{a}$ & $30.42 \mathrm{a}$ \\
\hline L88xL55 & $12.19 \mathrm{c}$ & $3.69 \mathrm{f}$ & $4.86 \mathrm{~b}$ & $0.55 \mathrm{~d}$ & $4,388.25 \mathrm{a}$ & $4,235.68 \mathrm{a}$ & $22.96 \mathrm{e}$ & $21.50 \mathrm{c}$ \\
\hline L88xL61 & $15.01 \mathrm{c}$ & $7.86 \mathrm{f}$ & $3.15 \mathrm{c}$ & $2.27 \mathrm{~d}$ & $4,316.98 \mathrm{a}$ & $4,305.62 \mathrm{a}$ & $22.50 \mathrm{e}$ & $24.34 \mathrm{~b}$ \\
\hline $\begin{array}{l}\text { L88xL70 } \\
\end{array}$ & $7.54 \mathrm{c}$ & $4.60 \mathrm{f}$ & $0.84 \mathrm{c}$ & $0.07 \mathrm{~d}$ & $3,106.65 b$ & $4,501.46 \mathrm{a}$ & $24.33 d$ & $22.58 \mathrm{~b}$ \\
\hline $\begin{array}{l}\text { L88xL76 } \\
\end{array}$ & $9.57 \mathrm{c}$ & $5.69 \mathrm{f}$ & $1.97 \mathrm{c}$ & $0.20 \mathrm{~d}$ & $4,433.02 \mathrm{a}$ & $4,647.67 \mathrm{a}$ & $24.63 \mathrm{~d}$ & $18.83 \mathrm{~d}$ \\
\hline L88xP1 & $18.79 \mathrm{~b}$ & $13.82 \mathrm{f}$ & $9.18 \mathrm{~b}$ & $2.76 \mathrm{~d}$ & $2,199.03 \mathrm{c}$ & $3,016.15 \mathrm{c}$ & $24.96 \mathrm{~d}$ & $22.96 \mathrm{~b}$ \\
\hline L88xP8 & $12.91 \mathrm{c}$ & $13.27 \mathrm{f}$ & $3.09 \mathrm{c}$ & $3.36 \mathrm{~d}$ & $3,046.57 \mathrm{~b}$ & $2,616.87 \mathrm{c}$ & $29.58 \mathrm{~b}$ & $23.02 \mathrm{~b}$ \\
\hline P1xL55 & $13.38 \mathrm{c}$ & $16.65 \mathrm{e}$ & $2.53 \mathrm{c}$ & $4.57 \mathrm{~d}$ & $3,910.11 \mathrm{a}$ & $3,876.68 \mathrm{~b}$ & $27.34 \mathrm{c}$ & $26.13 \mathrm{~b}$ \\
\hline $\begin{array}{l}\text { P1xL70 } \\
\end{array}$ & $19.79 \mathrm{~b}$ & $23.04 \mathrm{e}$ & $5.16 \mathrm{~b}$ & $3.86 \mathrm{~d}$ & $3,247.13 \mathrm{~b}$ & $3,019.11 \mathrm{c}$ & $28.08 \mathrm{c}$ & $30.63 \mathrm{a}$ \\
\hline P1x L77 & $12.88 \mathrm{c}$ & $21.00 \mathrm{e}$ & $5.84 \mathrm{~b}$ & $4.76 \mathrm{~d}$ & $2,432.02 \mathrm{c}$ & $3,158.94 \mathrm{c}$ & $27.04 \mathrm{c}$ & $30.59 \mathrm{a}$ \\
\hline P1xL61 & $15.56 \mathrm{c}$ & $45.00 \mathrm{~d}$ & $5.51 \mathrm{~b}$ & $8.24 \mathrm{c}$ & $2,635.48 \mathrm{c}$ & $3,353.60 \mathrm{~b}$ & $24.88 \mathrm{~d}$ & $31.33 \mathrm{a}$ \\
\hline P1xL76 & $16.05 \mathrm{c}$ & $51.75 \mathrm{c}$ & $5.72 \mathrm{~b}$ & $11.57 \mathrm{~b}$ & $3,513.57 \mathrm{~b}$ & $3,576.33 \mathrm{~b}$ & $29.38 b$ & $25.42 \mathrm{~b}$ \\
\hline P1xL88 & $16.70 \mathrm{c}$ & $14.02 \mathrm{f}$ & $5.37 \mathrm{~b}$ & $2.46 \mathrm{~d}$ & $3,341.14 \mathrm{~b}$ & $2,820.74 \mathrm{c}$ & $24.58 \mathrm{~d}$ & $22.09 \mathrm{c}$ \\
\hline P1xP8 & $14.26 \mathrm{c}$ & $45.26 \mathrm{~d}$ & $6,92 \mathrm{~b}$ & $7.75 \mathrm{c}$ & $3,208.88 \mathrm{~b}$ & $2,556.71 \mathrm{c}$ & $33.42 \mathrm{a}$ & $30.92 \mathrm{a}$ \\
\hline P8xL55 & $12.22 \mathrm{c}$ & $10.99 \mathrm{f}$ & $6.55 \mathrm{~b}$ & $0.98 \mathrm{~d}$ & $4,035.65 \mathrm{a}$ & $3,654.52 \mathrm{~b}$ & $32.54 \mathrm{a}$ & $23.00 \mathrm{~b}$ \\
\hline P8xL61 & $13.50 \mathrm{c}$ & $15.56 \mathrm{f}$ & $1.53 \mathrm{c}$ & $2.00 \mathrm{~d}$ & $4,767.01 \mathrm{a}$ & $3,832.40 \mathrm{~b}$ & $29.75 b$ & $31.92 \mathrm{a}$ \\
\hline P8xL70 & $16.23 \mathrm{c}$ & $16.32 \mathrm{e}$ & $6.51 \mathrm{~b}$ & $3.98 \mathrm{~d}$ & $3,382.14 b$ & $3,673.81 \mathrm{~b}$ & $32.71 \mathrm{a}$ & $30.25 \mathrm{a}$ \\
\hline P8xL76 & $6.45 \mathrm{c}$ & $25.47 \mathrm{e}$ & $3.79 \mathrm{c}$ & $2.47 \mathrm{~d}$ & $3,830.66 \mathrm{a}$ & $3,916.17 \mathrm{~b}$ & $33.08 \mathrm{a}$ & $26.54 \mathrm{~b}$ \\
\hline P8xL77 & $15.73 \mathrm{c}$ & $25.86 \mathrm{e}$ & $8.61 \mathrm{~b}$ & $4.54 \mathrm{~d}$ & $2,897.08 \mathrm{~b}$ & $4,538.59 \mathrm{a}$ & $25.92 \mathrm{c}$ & $30.22 \mathrm{a}$ \\
\hline P8xL88 & $13.02 \mathrm{c}$ & $14.19 \mathrm{f}$ & $4.38 \mathrm{c}$ & $1.48 \mathrm{~d}$ & $3,359.97 \mathrm{~b}$ & $3,230.11 \mathrm{c}$ & $28.17 \mathrm{c}$ & $23.08 \mathrm{~b}$ \\
\hline P8xP1 & $13.54 \mathrm{c}$ & $53.43 \mathrm{c}$ & $6.25 \mathrm{~b}$ & $13.98 \mathrm{a}$ & $3,001.18 \mathrm{~b}$ & $2,394.37 b$ & $33.87 \mathrm{a}$ & $31.59 \mathrm{a}$ \\
\hline L88 & $36.03 \mathrm{a}$ & $25.81 \mathrm{e}$ & $6.08 \mathrm{~b}$ & $3.69 \mathrm{~d}$ & $1,670.11 \mathrm{~d}$ & $2,031.41 \mathrm{c}$ & $18.50 \mathrm{f}$ & $15.75 \mathrm{~d}$ \\
\hline L77 & $28.29 \mathrm{a}$ & $30.89 \mathrm{e}$ & $5.43 \mathrm{~b}$ & $6.94 \mathrm{c}$ & $1,071.87 \mathrm{~d}$ & $1,035.91 \mathrm{~d}$ & $28.38 \mathrm{c}$ & $29.25 \mathrm{a}$ \\
\hline L55 & $34.79 \mathrm{a}$ & $75.78 \mathrm{a}$ & $16.09 \mathrm{a}$ & $17.40 \mathrm{a}$ & $1,521.88 \mathrm{~d}$ & $730.83 \mathrm{~d}$ & $27.31 \mathrm{c}$ & $20.50 \mathrm{c}$ \\
\hline L70 & $21.65 \mathrm{~b}$ & $52.59 \mathrm{c}$ & $5.14 \mathrm{~b}$ & $10.44 \mathrm{~b}$ & $1,638.48 \mathrm{~d}$ & $1,992.93 \mathrm{c}$ & $28.50 \mathrm{c}$ & $29.92 \mathrm{a}$ \\
\hline L61 & $28.42 \mathrm{a}$ & $61.33 \mathrm{~b}$ & $3.77 \mathrm{c}$ & $8.21 \mathrm{c}$ & $722.88 \mathrm{~d}$ & $475.26 \mathrm{~d}$ & $22.71 \mathrm{e}$ & $33.71 \mathrm{a}$ \\
\hline P1 & $20.53 \mathrm{~b}$ & $47.75 \mathrm{c}$ & $5.44 \mathrm{~b}$ & $8.69 \mathrm{c}$ & $1,284.83 \mathrm{~d}$ & $664.69 \mathrm{~d}$ & $28.83 \mathrm{c}$ & $29.58 \mathrm{a}$ \\
\hline L76 & $16.47 \mathrm{c}$ & $9.14 \mathrm{f}$ & $1.23 \mathrm{c}$ & $1.46 \mathrm{~d}$ & $1,633.28 \mathrm{~d}$ & $1,998.29 \mathrm{c}$ & $30.04 \mathrm{~b}$ & $23.00 \mathrm{~b}$ \\
\hline P8 & $20.52 \mathrm{~b}$ & $40.28 \mathrm{~d}$ & $4.00 \mathrm{c}$ & $6.04 \mathrm{c}$ & $1,814.40 \mathrm{~d}$ & $2,553.24 \mathrm{c}$ & $36.25 \mathrm{a}$ & $31.09 \mathrm{a}$ \\
\hline IAC 125 & $14.53 \mathrm{c}$ & $31.61 \mathrm{e}$ & $5.74 \mathrm{~b}$ & $8.04 \mathrm{c}$ & $3,215.19 b$ & $2,817.62 \mathrm{c}$ & $34.71 \mathrm{a}$ & $33.08 \mathrm{a}$ \\
\hline L70xL54 & $19.70 \mathrm{~b}$ & $60.65 \mathrm{~b}$ & $9.30 \mathrm{~b}$ & $12.3 \mathrm{~b}$ & $3,797.68 \mathrm{~b}$ & $3,355.37 \mathrm{~b}$ & $34.79 \mathrm{a}$ & $25.00 \mathrm{~b}$ \\
\hline P8xL54 & $14.24 \mathrm{c}$ & $40.85 \mathrm{~d}$ & $4.29 \mathrm{c}$ & $6.68 \mathrm{c}$ & $3,762.86 \mathrm{~b}$ & $3,205.60 \mathrm{c}$ & $32.79 \mathrm{a}$ & $31.92 \mathrm{a}$ \\
\hline UENF-14 & $16.90 \mathrm{c}$ & $20.70 \mathrm{e}$ & $4.21 \mathrm{c}$ & $3.28 \mathrm{~d}$ & $3,191.20 \mathrm{~b}$ & $3,778.18 \mathrm{~b}$ & $33.50 \mathrm{a}$ & $28.38 \mathrm{a}$ \\
\hline B. Viçosa & $15.07 \mathrm{c}$ & $10.14 \mathrm{f}$ & $3.84 \mathrm{c}$ & $2.61 \mathrm{~d}$ & $2,685.74 \mathrm{c}$ & $3,142.96 \mathrm{c}$ & $33.71 \mathrm{a}$ & $27.75 \mathrm{a}$ \\
\hline BRS Angela & $9.20 \mathrm{c}$ & $17.13 \mathrm{e}$ & $3.44 \mathrm{c}$ & $5.01 \mathrm{~d}$ & $2,560.12 \mathrm{c}$ & $2,768.61 \mathrm{c}$ & $34.38 \mathrm{a}$ & $28.83 \mathrm{a}$ \\
\hline
\end{tabular}

Means followed by the same letter in a column do not differ by the Scott-Knott test at a significance level of 5\%.

Genetics and Molecular Research 16 (3): gmr16039772 
For popping expansion in the first harvest, the genotypes were distributed into five groups, while four groups were formed in the second harvest. Within the groups formed in the first harvest, fifteen genotypes showed average estimates greater than $32.71 \mathrm{~mL} / \mathrm{g}$, composing the group with the best performance. Among these, parent P8 and controls L70 x L54 and IAC 125 had the best performance, with estimated popping expansion of $36.25,34.79$, and $34.71 \mathrm{~mL} / \mathrm{g}$, respectively. Regarding experimental hybrids, L76 x P8 expressed the highest popping expansion, $34.46 \mathrm{~mL} / \mathrm{g}$. However, the estimated grain yield was not sufficiently high: $3290.00 \mathrm{~kg} / \mathrm{ha}$; nevertheless, this magnitude exceeds by $9.67 \%$ the minimum recommended for a release, characterizing it as a hybrid that can be indicated for commercial crops.

In the second harvest, parents L61 and P8 had the best means composing the group of genotypes with the highest estimates for popping expansion: 33.71 and $31.09 \mathrm{~mL} / \mathrm{g}$, respectively. Controls IAC 125 and P8 x L54 had the best performance for this growing harvest, with estimates of 33.08 and $31.92 \mathrm{~mL} / \mathrm{g}$. These higher mean values of parent P8 in both growing harvests demonstrate its potential among the evaluated parents, characterizing it as promising to be used in breeding programs aimed at increasing the popping expansion. Experimental hybrids L61 x P8, P8 x P1, L77 x L61, and P1 x L77 had the best performances for popping expansion, with estimated values of $31.83,31.59,31.58$, and $31.33 \mathrm{~mL} / \mathrm{g}$, respectively.

The mean squares for GCA and SCA, in the second harvest, were significant for incidence and severity of E. turcicum, as well as for GY and PE, which indicates the existence of variability resulting from the action of additive and non-additive effects in the control of the gene expressions of these traits (Table 2). By contrast, for the first harvest, a significant effect of GCA was only observed for severity of E. turcicum, GY, and PE; for SCA, only for the incidence of leaves with symptoms of NLB. As also shown in Table 2, there was no significant reciprocal effect for the evaluated traits, either relating to resistance to NLB or for agronomic

\begin{tabular}{|c|c|c|c|c|c|}
\hline SV & d.f. & 1st harvest & 2 nd harvest & 1st harvest & 2 nd harvest \\
\hline Genotype & 63 & $27,454.8390^{* *}$ & $218,117.5369^{* *}$ & $4,468.2837 * *$ & $4,367.1203^{* *}$ \\
\hline GCA & 7 & $60,583.8321^{\dagger \mathrm{NS}}$ & $1,203,867.4560 * *$ & $18,258.8802 * *$ & $23,109.1557 * *$ \\
\hline SCA & 28 & $41,796.5796^{* *}$ & $179,669.4416^{* *}$ & $3,623.0738^{\mathrm{NS}}$ & $3,277.1100^{* *}$ \\
\hline Reciprocal & 28 & $4,830.8501^{\mathrm{NS}}$ & $10,128.1523^{\mathrm{NS}}$ & $1,865.8445^{\mathrm{NS}}$ & $771.6218^{\mathrm{NS}}$ \\
\hline Residual & 189 & $9,084.9527$ & $15,824.1628$ & $2,305.2210$ & 517.0652 \\
\hline \multicolumn{6}{|c|}{ Mean squares of effects } \\
\hline GCA & & 804.6700 & $18,563.1765$ & 249.2759 & 353.0014 \\
\hline SCA & & $8,177.9067$ & $40,961.3197$ & 329.4632 & 690.0112 \\
\hline Reciprocal & & -531.7628 & -712.0013 & -54.9221 & 31.8196 \\
\hline \multirow[t]{2}{*}{ SV } & d.f. & \multicolumn{2}{|c|}{ Grain yield } & \multicolumn{2}{|c|}{ Popping expansion } \\
\hline & & 1st harvest & 2 nd harvest & 1st harvest & 2 nd harvest \\
\hline Genotype & 63 & $3,662,308.1049 * *$ & $4,503,644.1416^{* *}$ & $46.6543^{* *}$ & $74.2646^{* *}$ \\
\hline GCA & 7 & $5,331,101.3828 * *$ & $10,253,968.3452^{* *}$ & $350.3801 * *$ & $613.2601 * *$ \\
\hline SCA & 28 & $6,354,133.1696^{* *}$ & $6,957,148.1128^{* *}$ & $6.9675^{\mathrm{NS}}$ & $8.9460 * *$ \\
\hline Reciprocal & 28 & $553,284.7207^{\mathrm{NS}}$ & $612,559.1194^{\mathrm{NS}}$ & $10.4096^{\mathrm{NS}}$ & $4.8343^{\mathrm{NS}}$ \\
\hline Residual & 189 & $710,220.2771$ & $458,242.0666$ & 6.5599 & 3.9158 \\
\hline \multicolumn{6}{|c|}{ Mean squares of effects } \\
\hline GCA & & $72,201.2673$ & $153,058.2231$ & 5.3722 & 9.5210 \\
\hline SCA & & $1,410,978.2231$ & $1,624,726.5115$ & 0.1019 & 1.2576 \\
\hline Reciprocal & & $-19,616.9445$ & $19,289.6316$ & 0.4812 & 0.1148 \\
\hline
\end{tabular}

NS, not significant at the 0.05 probability level. **Significant at the 0.01 probability level. SV: source of variation; d.f.: degrees of freedom.

Genetics and Molecular Research 16 (3): gmr16039772 
attributes, which suggests that the performance of the parent as a pollen donor or receptor can be the same. Thus, resistance to NLB cannot be superior if the crosses are inverted, which is an important fact in breeding programs to prevent additional work and costs in potentiating variability through hybridization.

Non-additive genetic effects predominated in the evaluated traits (Table 2), except popping expansion, which determines that hybridization is the best alternative to obtain gains in breeding aimed at resistance to E. turcicum and grain yield. Results concerning the prominence of gene effects on resistance to E. turcicum in conventional corn have been controversial. Paterniani et al. (2000) and Vivek et al. (2010), for instance, found that the additive genes were the most important in the expression of resistance to E. turcicum; the opposite occurred in the study developed by Nihei and Ferreira (2012). These findings indicate that genetic control for this trait may vary across the different sources of resistance. In this case, it is up to breeding programs to characterize their sources of resistance to achieve maximum efficiency during the selection process (Lopes et al., 2007).

For grain yield, the mean squares of the effects associated with GCA were lower than those associated with SCA, demonstrating the higher importance of non-additive concerning additive effects in the genetic control of this trait. Analogously to these results, superior significant SCA effects concerning GCA were found by Pereira and Amaral Júnior (2001) and Freitas Jr et al. (2006) in popcorn.

As for popping expansion, the additive genetic effect was the most important; therefore, intrapopulation methods are the most recommended for obtaining gains in this trait. These results agree with those obtained by da Silva et al. (2010), Vieira et al. (2011), and Cabral et al. (2015).

In the first harvest, parents L76, L61, and L70 presented negative GCA estimates for incidence and severity of E. turcicum (Table 3 ), and are thus recommended for the generation of superior hybrids. In the second harvest, negative GCA estimates for incidence and severity of E. turcicum were expressed by parents L88, P8, L77, and L76. Only L76 displayed a negative estimate of GCA for incidence and severity of E. turcicum in both growing harvests (Table 3); moreover, according to the Scott-Knott test, L76 was allocated in the group with the greatest resistance to both variables related to NLB (Table 1). Therefore, L76 can be considered a line of interest for participation in breeding programs aimed at the production of segregants with resistance to NLB, especially in the composition of hybrids, given the greater evidence of dominance in the expression of resistance.

Table 3. Estimates of general combining ability, evaluated in a full diallel with eight parents, in the first harvest (October 2014 to January 2015) and the second harvest (May to August 2015) in Campos dos Goytacazes, RJ, Brazil.

\begin{tabular}{l|c|c|c|c|c|c|c|c}
\hline \multirow{2}{*}{ Parents } & \multicolumn{2}{|c|}{ Incidence of NLB } & \multicolumn{2}{c|}{ Severity of NLB } & \multicolumn{2}{c|}{ Grain yield } & \multicolumn{2}{c}{ Popping expansion } \\
\cline { 2 - 9 } & $\begin{array}{c}\text { 1st } \\
\text { harvest }\end{array}$ & $\begin{array}{c}2 \mathrm{nd} \\
\text { Harvest }\end{array}$ & $\begin{array}{c}\text { 1st } \\
\text { harvest }\end{array}$ & $\begin{array}{c}2 \mathrm{nd} \\
\text { Harvest }\end{array}$ & $\begin{array}{c}\text { 1st } \\
\text { harvest }\end{array}$ & $\begin{array}{c}2 \mathrm{nd} \\
\text { harvest }\end{array}$ & $\begin{array}{c}\text { st } \\
\text { harvest }\end{array}$ & $\begin{array}{c}2 \mathrm{nd} \\
\text { harvest }\end{array}$ \\
\hline L88 & 41.5590 & -107.4250 & -7.4022 & -17.8440 & 431.5150 & 403.6550 & -3.6400 & -5.4520 \\
\hline P8 & -12.3813 & -33.1384 & 3.4578 & -0.6050 & -17.8590 & 67.3220 & 1.3740 & 2.2060 \\
\hline L61 & -21.0606 & 118.0447 & -17.9584 & 7.8530 & -300.2580 & -87.3050 & 0.0250 & 3.6650 \\
\hline L70 & -9.7463 & 103.6778 & -1.4622 & 12.8540 & -24.5870 & 358.4060 & 0.5940 & 1.5500 \\
\hline L77 & 3.5888 & -169.1720 & -6.2791 & -21.6050 & 79.5770 & 398.5200 & 4.1840 & 0.9600 \\
\hline L55 & 50.7094 & 194.2666 & 36.7572 & 31.0370 & -456.1800 & -639.4360 & -2.2100 & -2.7810 \\
\hline P1 & -15.4450 & 52.5791 & 6.1866 & 9.7040 & -14.1570 & -516.9830 & -0.6070 & 1.9250 \\
\hline L76 & -37.2169 & -158.8330 & -13.2997 & -21.3940 & 301.9490 & 37.44900 & 0.2790 & -2.0720 \\
\hline
\end{tabular}


Contrary to what has been considered for disease-related traits, in the evaluation of grain yield, higher positive estimates of GCA are preferred since the effects of allelic complementation are predominant in the generation of superior genotypes. For this trait, in the first harvest, the highest GCA estimates were observed in parent L88, followed L76 and L77. Of these, L88 and L76, together with L70, were the lines that stood out, also in the second harvest, for revealing high positive GCA estimates. Thus, for both growing harvests, L88 and L76 can be considered the parents of interest for obtaining gains in grain yield.

Regarding popping expansion, in the first harvest, the lines L61, P8, P1, and L70 showed the most significant positive estimates for GCA, whereas in the second harvest the lines L77, P8, and L70 were superior. For popcorn breeding programs, genotypes that conciliate favorable genes for GY and PE should be prioritized, because these are the characteristics of highest economic importance, besides other traits that may make the genotype superior, of course. In this context, most of the parents that were highlighted for GY were not for PE. In popcorn breeding, the existence of a negative correlation between PE and GY complicates the selection of genotypes for these two traits of economic value (Daros et al., 2004). For this reason, none of the parents that were superior for PE and GY could be simultaneously highlighted.

In the first harvest, 17 hybrid combinations were noteworthy for their negative SCA estimates, both incidence and severity of NLB. In the second harvest, however, 17 hybrids displayed negative SCA estimates for both traits related to resistance to NLB (Table 4). The superior hybrids - for showing negative estimates for incidence and severity of NLB - common for both growing harvests were L88 x L61, L88 x L70, L88 x L55, P8 x L70, L61 x L77, L61 x P1, L70 x L77, L77 x P1, and L55 x L76, which characterizes them as of interest when aiming at reduced attack of NLB in crops.

For grain yield, in both growing conditions, a high number of combinations with positive SCA estimates were obtained. The three combinations that most stood out in both harvests were L61 x L77, L61 x L76, and L77 x L76, whose means were higher than $3900.00 \mathrm{~kg} / \mathrm{ha}$.

The SCA for popping expansion in the first harvest revealed that 17 combinations presented positive magnitudes. However, combinations L76 x P8, P8 x P1, L70 x P8, P8 x L77, P8 x L55, P8 x L61, L76 x L70, and L77 x L76 can be considered promising for that trait, because in addition to revealing positive values for SCA they also showed satisfactory means for the trait (Table 1) and originated from at least one parent with the potential to increase popping expansion. This demonstrates that the desirable effect of the genetic accumulation of parents L70, L77, and P8 was translated into a satisfactory effect of genetic complementation in the combinations.

In the second harvest, 15 combinations expressed positive SCA estimates for popping expansion, in which P8 x L61, L77 x P8, L77 x L70, L61 x L77, L61 x L76, L70 x P8, and L61 x L70 were noteworthy. In this regard, P8 x L61 showed a higher SCA estimate than the other selected combinations, averaging $31.92 \mathrm{~mL} / \mathrm{g}$ (Table 1), thus proving to be of great potential to increase PE. Despite the difficulty gathering high yields and good popping expansion in one hybrid, L61 x L77 managed to comprise quality with productivity and parent L61 had the highest GCA for popping expansion, while L77 expressed a good GCA estimate for grain yield. This denotes that the hybrid responded as expected based on the parental GCA. This was not true for the other combinations, whose hybrids, despite originating from GCA desirable for PE and GY, did not display good allelic complementation for both traits together.

Given the results, promising hybrids can be indicated to increase GY and PE and reduce incidence and severity of NLB. In this context, in the first harvest, combination P8

Genetics and Molecular Research 16 (3): gmr16039772 


\begin{tabular}{|c|c|c|c|c|c|c|c|c|}
\hline \multirow{2}{*}{$\begin{array}{l}\text { Effects } \\
\left(\hat{\mathrm{s}}_{\mathrm{ii}}\right) \text { and }\left(\hat{\mathrm{s}}_{\mathrm{ij}}\right)\end{array}$} & \multicolumn{2}{|c|}{ Incidence of NLB } & \multicolumn{2}{|c|}{ Severity of NLB } & \multicolumn{2}{|c|}{ Grain yield } & \multicolumn{2}{|c|}{ Popping expansion } \\
\hline & 1st harvest & 2 nd harvest & 1st harvest & 2 nd harvest & 1st harvest & 2 nd harvest & 1st harvest & 2 nd harvest \\
\hline L88 x L88 & 215.0313 & 257.5809 & 38.0791 & 34.3783 & $-2,555.5260$ & $-2,275.2420$ & -2.2000 & 0.1320 \\
\hline L88 x P8 & -1.1456 & 5.3247 & 28.0791 & 6.2695 & 319.9320 & -85.7690 & -0.8980 & -0.0370 \\
\hline L88 x L61 & -92.7863 & -109.096 & -11.9997 & -13.8836 & 113.9720 & 673.4230 & 0.9710 & -1.5140 \\
\hline L88 x L70 & -62.2206 & -113.0770 & -19.8709 & -15.4448 & 637.4010 & 929.1820 & 1.4610 & -0.3290 \\
\hline $\mathrm{L} 88 \times \mathrm{L} 77$ & -15.6306 & 36.1484 & -5.8841 & 2.6739 & 697.9720 & 301.2370 & 0.3510 & -0.2260 \\
\hline L88 x L55 & -0.8013 & -108.5050 & -2.6953 & -25.2373 & 156.7640 & 53.3890 & 0.3510 & -0.2260 \\
\hline L88 x P1 & -37.7619 & -13.4978 & -24.6947 & 1.2708 & 730.7810 & 370.8370 & 0.1030 & 2.7970 \\
\hline L88 x L76 & -4.6850 & 81.1841 & -1.0134 & 9.9733 & -101.2950 & 32.9430 & 0.1510 & -0.4700 \\
\hline P8 x P8 & 79.5575 & 288.9984 & -10.3509 & 14.4808 & $-1,512.4900$ & $-1,080.7460$ & -0.6860 & 0.6240 \\
\hline P8 x L61 & -50.0981 & 12.4703 & -6.0397 & 6,7227 & 788.0950 & 253.8720 & 0.1850 & 1.6700 \\
\hline P8 x L70 & -32.1875 & -48.3228 & $\begin{array}{l}-7.2459 \\
\end{array}$ & -5.9486 & 265.4140 & 323.4450 & 0.0650 & -0.1250 \\
\hline P8 x L77 & 0.1975 & -22.6628 & -8.4891 & -2.1348 & 510.9650 & 379.7000 & -0.6900 & -1.1450 \\
\hline P8 x L55 & 0.5769 & -177.2770 & 8.9347 & -18.4211 & -653.3730 & 124.2020 & 0.3100 & 0.6500 \\
\hline P8 x P1 & 39.9713 & -60.9691 & 13.1853 & 3.4470 & 166.4190 & 55.7100 & 1.0450 & -0.4150 \\
\hline P8 x L76 & -36.8719 & 2.4378 & -18.0734 & -4.4155 & 115.0380 & 29.5870 & -0.7900 & -0.1650 \\
\hline L61 x L61 & 214.7563 & 308.2822 & 27.4616 & 19.1445 & $-2,039.2110$ & $-2,849.4710$ & 0.3520 & 0.8100 \\
\hline L61 x L70 & 86.5219 & 166.8991 & 16.3003 & 30.3233 & -520.0560 & -920.9020 & -2.0200 & 0.0850 \\
\hline L61 x L77 & -81.1131 & -180.5360 & -11.0328 & -21.6680 & 347.1040 & 748.0020 & -3.7300 & -0.1000 \\
\hline L61 x L55 & -14.3988 & 28.1853 & -10.7241 & 7.4708 & 311.0810 & 666.3000 & 1.0200 & -0.0400 \\
\hline L61 x P1 & -53.5494 & -128.1520 & -6.6784 & -19.9561 & 512.2280 & 661.5370 & -0.2920 & -1.1100 \\
\hline L61 x L76 & -9.3325 & -61.9903 & 2.7128 & -8.1536 & 486.7870 & 767.2390 & 0.4220 & 1.3090 \\
\hline L70 x L70 & 112.5275 & 218.8959 & 24.6791 & 31.9420 & $-1,674.9520$ & $-2,223.2230$ & $\begin{array}{l}-0.6670 \\
\end{array}$ & 0.3000 \\
\hline L70 x L77 & -73.2275 & -187.8740 & -21.9941 & -26.8542 & 779.2940 & 717.6360 & -0.5800 & -0.1700 \\
\hline L70 x L55 & -52.2681 & 33.9322 & -26.5653 & 2.1545 & 498.2100 & 225.3480 & -0.9600 & 0.2100 \\
\hline L70 x P1 & 8.8563 & -47.9003 & 13.6653 & -13.0373 & 4.0520 & 476.3210 & -1.1710 & -1.2000 \\
\hline L70 x L76 & 11.9981 & -22.5534 & 21.0316 & -3.1348 & 10.6370 & 472.1930 & -0.5820 & 0.5990 \\
\hline L77 x L77 & 176.4175 & 480.7459 & 27.5328 & 68.9695 & $-2,449.8910$ & $-2,543.1350$ & -0.0970 & 0.1560 \\
\hline L77 x L55 & -7.5481 & -155.9180 & 8.7216 & -25.4217 & -215.9190 & 18.6680 & 02860 & -0.5170 \\
\hline L77 x P1 & -7.4138 & -24.8153 & -6.1428 & -3.5486 & -224.7570 & -98.7200 & 0.0290 & -1.8210 \\
\hline L77 x L76 & 8.3181 & 54.9116 & 17.2884 & 7.9839 & 555.2320 & 476.5920 & 1.2030 & 0.6020 \\
\hline L55 x L55 & 173.1763 & 361.7584 & 75.6003 & 52.7870 & -928.3670 & $-1,489.6400$ & -0.8500 & -0.1400 \\
\hline L55 x P1 & -46.2844 & 113.4259 & -13.6641 & 16.9052 & 212.7600 & 132.6180 & 1.5930 & 1.4700 \\
\hline L55 x L76 & -52.4525 & -95.6022 & -39.6078 & -10.2373 & 618.8440 & 269.0950 & -0.8390 & -0.9460 \\
\hline P1 x P1 & 99.8650 & 233.5934 & -1.8184 & 26.9633 & $-2,049.4630$ & $-1,800.6850$ & 0.5450 & -0.4590 \\
\hline P1 x L76 & -3.6831 & -71.6847 & 26.1478 & -12.0442 & 647.9810 & 202.3820 & -1.1860 & 0.4850 \\
\hline L76 x L76 & 86.7088 & 113.2972 & -8.4859 & 20.0283 & $-2,333.2250$ & $-2,250.0310$ & 0.2930 & -0.7920 \\
\hline \multicolumn{9}{|c|}{ Reciprocal } \\
\hline L61 x L88 & 24.5400 & 75.1600 & 3.8450 & 3.2750 & -510.3750 & -3.6650 & -1.3750 & 2.8150 \\
\hline L70 x L88 & -10.8600 & 34.6050 & -1.1700 & 3.8550 & 95.0950 & 220.6450 & -2.0650 & 0.2900 \\
\hline L77 x L88 & -11.2850 & -29.1700 & 2.7100 & -1.1950 & -76.3300 & -136.2950 & 0.7050 & -0.0300 \\
\hline L55 x L88 & -14.5250 & 20.4750 & -6.5350 & 10.3050 & -247.5750 & -297.8400 & 0,3100 & -0.0400 \\
\hline P1 x L88 & -3.6300 & -38.5450 & -3.7250 & -12.7600 & 256.2650 & 75.5500 & -0.8750 & 0.4150 \\
\hline L76 x L88 & -45.7850 & 17.4550 & -10.2500 & 4.8150 & 349.1350 & -636.2100 & 0.1900 & 0.4350 \\
\hline L61 x P8 & -4.8150 & 7.0450 & -13.7750 & 3.5400 & -77.0050 & -0.8800 & 0.7280 & -1.6580 \\
\hline $\mathrm{L} 70 \times \mathrm{P} 8$ & -2.7100 & 14.2150 & -19.5150 & 0.5700 & 40.1250 & -37.8850 & 0.4480 & 0.1270 \\
\hline L77 x P8 & 15.9700 & 0.3950 & 1.9950 & 7.6950 & 381.6900 & 319.4050 & 0.2330 & 1.0300 \\
\hline L55 x P8 & -55.2400 & 5.9500 & -25.9850 & -10.5500 & 196.8250 & 107.5100 & -0.7430 & 0.8670 \\
\hline P1 x P8 & 3.0200 & -61.5100 & 4.1850 & -16.4050 & -114.8700 & 568.4200 & 0.3800 & -0.1630 \\
\hline L76 x P8 & 17.3650 & 4.5950 & 4.6400 & 2.4450 & -257.1550 & -227.1800 & 0.5380 & -0.7890 \\
\hline L70 x L61 & -62.4300 & 5.2500 & -8.1050 & -11.4000 & -40.0050 & -44.1700 & -0.0780 & 1.1350 \\
\hline L77 x L61 & 8.4000 & -35.7850 & -3.3750 & -2.3600 & -382.3800 & 301.5700 & -2.5380 & 0.6330 \\
\hline L55 x L61 & 6.8250 & -55.1250 & 11.3000 & -13.5000 & -281.7700 & -85.3000 & 0.4360 & 0.3950 \\
\hline P1 x L61 & -14.8300 & -17.9400 & 12.2250 & -15.2500 & 270.2400 & 359.5800 & 0.8550 & -0.6700 \\
\hline L76 x L61 & -32.3750 & -64.0500 & -11.8800 & -9.7050 & -237.9150 & 162.9250 & 1.6650 & 0.1250 \\
\hline L77 x L70 & 12.1200 & 1.7500 & 5.4600 & -0.7350 & 236.1300 & 32.4350 & 0.5330 & 0.1430 \\
\hline L55 x L70 & 38.6300 & 53.2450 & 15.7350 & 14.1450 & 133.5200 & 132.6700 & 0.0560 & -0.7750 \\
\hline P1 x L70 & 36.7500 & -65.9750 & 48.7050 & -11.4400 & -430.8350 & 721.5050 & 0.6650 & -0.7500 \\
\hline L76 x L70 & 18.9100 & -9.4100 & 4.6450 & 4.1350 & -180.3650 & $-83,4000$ & 0.1900 & -0.0350 \\
\hline L55 x L77 & -21.4350 & -1.4450 & -22.8850 & 0.7300 & 571.0550 & -97.7050 & -0.4900 & 0.0450 \\
\hline P1 x L77 & -8.1850 & 9.1400 & 8.4100 & -10.2300 & 156.0700 & 306.6200 & 0.9550 & -1.1250 \\
\hline L76 x L77 & 2.0550 & 7.3950 & -18.0350 & -2.7550 & -88.8850 & 154.5950 & 0.2250 & 0.3350 \\
\hline P1 x L55 & -12.4050 & 44.1900 & -1.3350 & 26.6050 & -103.8500 & -81.1700 & 0.4150 & 1.1250 \\
\hline L76 x L55 & -22.3550 & 32.5500 & -1.3650 & -4.5450 & -82.8900 & -373.1900 & 0.3300 & -0.1650 \\
\hline L76 x P1 & -13.4900 & -10.5900 & -30.2500 & 5.7150 & 262.7300 & -95.2900 & -0.8750 & 0.0200 \\
\hline
\end{tabular}

Genetics and Molecular Research 16 (3): gmr16039772 
x L61 expressed the highest estimate for both traits together, whereas in the second harvest, combinations L61 x L76 and L61 x L77 stood out for the elevated negative SCA estimates for incidence and severity of NLB as well as high positive estimates for GY and PE.

\section{Conflicts of interest}

The authors declare no conflict of interest.

\section{ACKNOWLEDGMENTS}

The authors thank the Foundation for Research Support of the State of Rio de Janeiro (FAPERJ) and the National Council for Scientific and Technological Development (CNPq) for the financial support to the experiment, and the Coordination of Improvement of Higher Education Personnel (CAPES), for granting a doctoral scholarship to J.S. Santos.

\section{REFERENCES}

Agrios GN (1988). Plant Pathology. 3rd edn. Academic Press, San Diego.

Agroceres (1996). Guia Agroceres de sanidade. Sementes Agroceres, São Paulo.

Arnhold E (2008). Seleção para resistência a doenças foliares em famílias S1 de milho-pipoca. Rev. Ceres 55: 89-93.

Bergamin Filho A and Amorim JAM (2011). Manejo integrado de doenças. In: Manual de Fitopatologia: Princípios e conceitos (Amorim L, Rezende JAM and Bergamin Filho, eds.). 4th edn. Ceres, São Paulo.

Cabral PD, Amaral Jr AT, Viana AP, Vieira HD, et al. (2015). Combining ability between tropical and temperate popcorn lines of seed quality and agronomic traits. Aust. J. Crop Sci. 9: 256-263.

Carson ML (1995). Inheritance of latent period length in maize infected with Exserohilum turcicum. Plant Dis. 79: 581585. https://doi.org/10.1094/PD-79-0581

Costa RV, Casela CR and Cota LV (2009). Cultivo do Milho: Doenças. Available at [http://www.cnpms.embrapa.br/ publicacoes/milho_5_ed/doencas.htm]. Accessed January 15, 2016.

Cruz CD (2013). GENES: a software package for analysis in experimental statistics and quantitative genetics. Acta Sci. Agron. 35: 271-276. https://doi.org/10.4025/actasciagron.v35i3.21251

Cruz CD, Regazzi AJ and Carneiro PC (2012). Modelos biométricos aplicados ao melhoramento genético. 4th edn. UFV, Viçosa.

da Silva VQ, do Amaral Júnior AT, Gonçalves LSA, Freitas Júnior SP, et al. (2010). Combining ability of tropical and temperate inbred lines of popcorn. Genet. Mol. Res. 9: 1742-1750. https://doi.org/10.4238/vol8-3gmr900

Daros M, Amaral Júnior AT, Pereira MG, Santos FS, et al. (2004). Correlações entre caracteres agronômicos em dois ciclos de seleção recorrente em milho-pipoca. Cienc. Rural 34: 1389-1394. https://doi.org/10.1590/S0103$\underline{84782004000500010}$

Embrapa (2008). Comportamento das cultivares de milho disponíveis no mercado brasileiro na safra 2007/08 em relação às principais doenças. Embrapa Milho e Sorgo, Sete Lagoas. Available at [http://www.cnpms.embrapa.br/milho/ cultivares/tabela2.htm]. Accessed February 24, 2016.

Fantin GM, Sawazaki E and Barros BC (1991). Avaliação de genótipos de milho pipoca quanto à resistência a doenças e qualidade da pipoca. Summa Phytopathol. 17: 90-99.

Freitas Jr SP, Amaral Júnior AT, Pereira MG, Cruz CD, et al. (2006). Capacidade combinatória em milho pipoca por meio de dialelo circulante. (In Portuguese, with English abstract.). Pesqui. Agropecu. Bras. 41: 1599-1607. https://doi. org/10.1590/S0100-204X2006001100005

Griffing BA (1956). Concept of general and specific combining ability in relation to diallel crossing systems. Aust. J. Biol. Sci. 9: 463-493. https://doi.org/10.1071/BI9560463

Ishfaq A, Dar ZA, Lone AA, Ali G, et al. (2014). Disease reaction studies of maize against turcium leaf blight involving indigenous cytosterile source. Afr. J. Microbiol. Res. 27: 2592-2597.

Hallauer AR (2001). Specialty corns. CRC Press. Boca Raton, London, New York, and Washington.

Hallauer AR, Carena JM and Miranda Filho JB (2010). Quantitative genetics in maize breeding. Springer, New York, 477-459.

Genetics and Molecular Research 16 (3): gmr16039772 
Hoseney RC, Zeleznak K and Abdelrahman A (1983). Mechanism of Popcorn popping. J. Cereal Sci. 1: 43-52. https://doi. org/10.1016/S0733-5210(83)80007-1

Larish LLB and Brewbaker JL (1999). Diallel analyses of temperate and tropical popcorns. Maydica 44: 279-284.

Leonello LAF, Cazetta DA and Fornasieri Filho D (2009). Características agronômicas e qualidade comercial de cultivares de milho pipoca em alta população. Acta Sci. Agron. 31: 215-220.

Lopes MTG, Lopes R, Brunelli KR, Silva HP, et al. (2007). Controle genético da resistência à mancha-de-Phaeosphaeria em milho. Cienc. Rural 37: 605-611. https://doi.org/10.1590/S0103-84782007000300001

Miranda GV, Souza LV, Fidélis RR, Godoy CL, et al. (2002). Reação de cultivares de milho-pipoca à helmintosporiose. Rev. Ceres 49: 513-521.

Miranda GV, Coimbra RR, Godoy CL, Souza LV, et al. (2003). Potencial de melhoramento e divergência genética de cultivares de milho pipoca. Pesqui. Agropecu. Bras. 38: 681-688. https://doi.org/10.1590/S0100-204X2003000600003

Moterle LM, Braccini AL, Scapim CA, Pinto RJB, et al. (2012). Combining ability of popcorn lines for seed quality and agronomic traits. Euphytica 185: 337-347. https://doi.org/10.1007/s10681-011-0458-2

Nihei TH and Ferreira JM (2012). Análise dialélica de linhagens de milho com ênfase na resistência a doenças foliares. Pesqui. Agropecu. Bras. 47: 369-377. https://doi.org/10.1590/S0100-204X2012000300008

Pajic Z, Eric U, Srdic J, Mladenovic Drinic S, et al. (2008). Popping volume and grain yield in diallel set of popcorn inbred lines. Genetika 40: 249-260. https://doi.org/10.2298/GENSR0803249P

Paterniani E and Miranda Filho JB (1978). Melhoramento de populações. In: Melhoramento e produção de milho no Brasil (Paterniani E, ed.). ESALQ, Piracicaba, 202-246.

Paterniani MEAGZ, Sawazaki E, Dudienas C, Duarte AP, et al. (2000). Diallel crosses among maize lines with emphasis on resistance to foliar diseases. Genet. Mol. Biol. 23: 381-385. https://doi.org/10.1590/S1415-47572000000200024

Pereira MG and Amaral Júnior AT (2001). Estimation of genetic components in popcorn based on nested design. Crop Breed. Appl. Biotechnol. 71: 3-10. https://doi.org/10.13082/1984-7033.v01n01a01

Pinto RJB, Kvitschal MV, Scapim CA, Fracaro M, et al. (2007). Análise dialélica parcial de linhagens de milho-pipoca. Rev. Bras. Milho Sorgo 6: 325-337. https://doi.org/10.18512/1980-6477/rbms.v6n3p325-337

Sabato EO and Pinto NFJA (2013). Identificação e Controle de Doenças na Cultura do Milho. 2nd edn. EMBRAPA.

Silva WJ, Vidal BC, Pereira AC, Zerbeto M, et al. (1993). What makes popcorn pop? Nature 362: 417-417. https://doi. org $/ 10.1038 / 362417 \mathrm{a} 0$

Smith DR and White DG (1988). Diseases of corn. In: Corn and Corn Improvement (Sprague GF and Dudley JW, eds.). Agronomy 18: 687-766.

Viana JMS, Valente MSF, Scapim CA, Resende MDV, et al. (2011). Genetic evaluation of tropical popcorn inbred lines using BLUP. Maydica 56: 273-281.

Vieira RA, Tessmann DJ, Hata FT, Souto ER, et al. (2009). Resistência de híbridos de milho-pipoca a Exserohilum turcicum, agente causal da helmintosporiose do milho. Sci. Agrar. 10: 391-395. https://doi.org/10.5380/rsa.v10i5.15196

Vieira RA, Scapim CA, Tessmann DJ and Hata FT (2011). Diallel analysis of yield, popping expansion, and southern rust resistance in popcorn lines. Rev. Cienc. Agron. 42: 774-780. https://doi.org/10.1590/S1806-66902011000300025

Vieira RA, Mesquini RM, Silva CN, Hata FT, et al. (2013). A new diagrammatic scale for the assessment of northern corn leaf blight. Crop Prot. 56: 55-57. https://doi.org/10.1016/j.cropro.2011.04.018

Vivek B, Odongo O, Njuguna J, Imanywoha J, et al. (2010). Diallel analysis of grain yield and resistance to seven diseases of African maize (Zea Mays L.) inbred lines. Euphytica 172: 329-340. https://doi.org/10.1007/s10681-009-9993-5

Genetics and Molecular Research 16 (3): gmr16039772 